\title{
Takotsubo cardiomyopathy in a young woman with severe adrenal insufficiency and hypothyroidism (EP-1311)
}

\section{Aleksandra Kruszyńska'1, Jadwiga Słowińska-Srzednicka', Agata Popielarz-Grygalewicz2,3}

1Department of Endocrinology, The Medical Centre of Postgraduate Education, Warsaw, Poland

2Department of Cardiology, Bielanski Hospital, Warsaw, Poland

${ }^{3}$ Department of Cardiology, Faculty of Physiotherapy, Warsaw Medical University, Warsaw, Poland

\section{INTRODUCTION:}

Takotsubo Cardiomyopathy (TTC) is a reversible cardiomyopathy induced by severe emotional stress or severe clinical conditions. It is characterized by transient left ventricular systolic dysfunction and electrocardiographic changes. This cardiomyopathy has been described in some severe neurological conditions and in patients with pheochromocytoma. To date, some cases of this cardiomyopathy have been described in patients with hyponatremia due to isolated secondary adrenal insufficiency or in a patient with both hypothyroidism and adrenal insufficiency. TTC has been known to be more prevalent in women (the role of sex and hormones is not yet well defined). Adrenocortical failure is considered to be one of the triggering factors for ampulla cardiomyopathy.

\section{CASE REPORT:}

43-year-old woman was admitted to our Endocrinology Department with severe hypothyroidism, asthenia without syncope, weight loss ( $26 \mathrm{~kg}$ over the previous 4 months) and amenorrhea (her last period was 6 months before admission). The patient's general complaint was lack of strength and tiredness and she reported abdominal pain. She presented with asthenia,

normal $\mathrm{BMl}=22 \mathrm{~kg} / \mathrm{m}^{2}$, pale skin, bradycardia, low normal blood pressure. Her sexual hair was normal; the thyroid was enlarged and stiff on physical examination.

The endocrinological and biochemical laboratory data are presented in the Table 1

On admission, her plasma cortisol was $0 \mathrm{mcg} / \mathrm{dl}$. Blood glucose levels were of $70-76 \mathrm{mg} / \mathrm{dl}$. Urine collection for metanephrines was performed twice and was within the normal range, but the first obtained result (in the state of adrenal insufficiency) was more than twofold higher (= 508 $\mathrm{mcg} / \mathrm{d}$, normal: $100-1000 \mathrm{mcg} / \mathrm{d})$ than the second one (= $209 \mathrm{mcg} / \mathrm{d})$ after the patient had received adequate substitution with hydrocortisone.

An echocardiography was performed and revealed ampulla cardiomyopathy (Figure 1a).

Electrocardiography showed T wave inversion in almost all leads (Figure 2a).

Two weeks after treatment with hydrocortisone and thyroxine, an echocardiographic examination showed recovered left ventricular wall motion and improvement in the ejection fraction and electrocardiography was normal (Figures $1 b, 2 b)$.

Adrenal MRI was unremarkable. All other abdominal organs were normal, there were no paragangliomas detected on MRI.
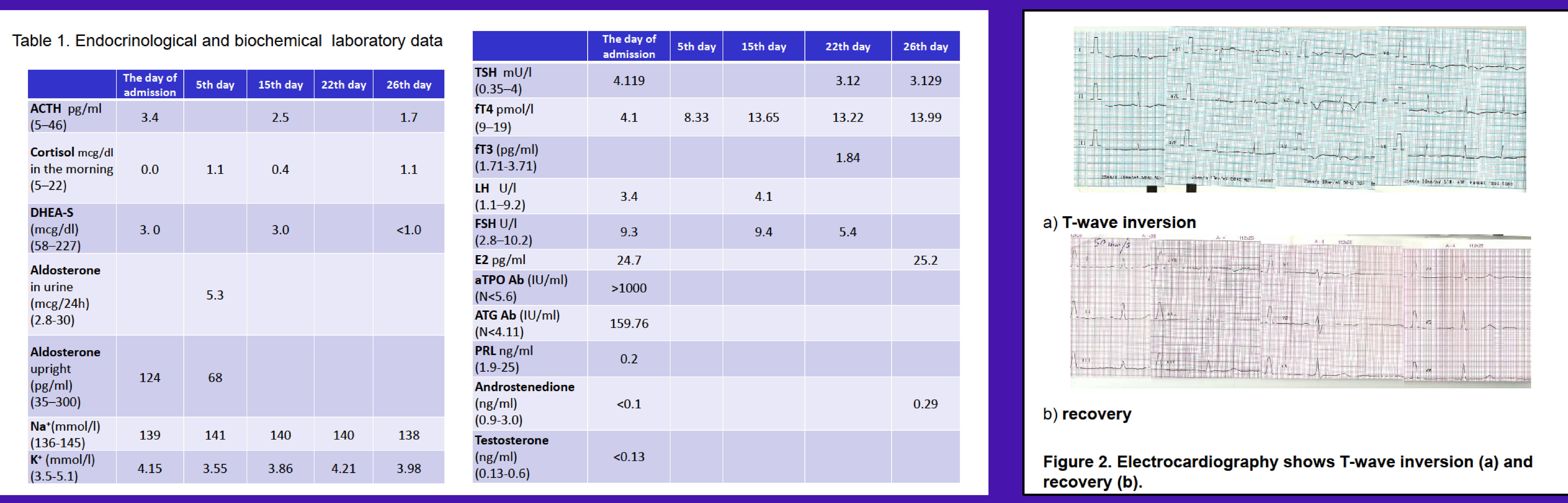

CONCLUSIONS: This is, to our knowledge, the first report of TTC due to hypothyroidism and adrenal insufficiency with no hyponatremia, no evident hypoglycemia and no evident catecholamine overproduction in a patient with hypopituitarism with secondary hypothyroidism, secondary adrenal insufficiency and hypogonadism as well as with primary hypothyroidism due to Hashimoto's thyroiditis.

Adrenocortical failure is considered to be one of the triggering factors for ampulla cardiomyopathy.

All patients with severe adrenal insufficiency should be screened for cardiomyopathy and first-line treatment in these cases consists of proper steroid substitution and careful monitoring of cardiac function. 